\title{
Failure and Ejection Behavior of Concrete Materials under Internal Blast
}

\author{
Haifu Wang, Jianguang Xiao, Yuanfeng Zheng, and Qingbo Yu \\ State Key Laboratory of Explosion Science and Technology, Beijing Institute of Technology, Beijing 100081, China
}

Correspondence should be addressed to Haifu Wang; wanghf@bit.edu.cn

Received 25 August 2015; Accepted 8 December 2015

Academic Editor: Isabelle Sochet

Copyright (C) 2016 Haifu Wang et al. This is an open access article distributed under the Creative Commons Attribution License, which permits unrestricted use, distribution, and reproduction in any medium, provided the original work is properly cited.

In order to investigate the failure and ejection behavior of concrete materials under internal blast, the default Riedel-HiermaierThoma (RHT) concrete model in AUTODYN and a meshfree processor called SPH are employed in this numerical simulation. It is shown that the failure mechanisms are significantly different in these damaged zones. Crushed zone is caused by shear failure while fractured zone is induced by tensile failure, and spalled zone is formed by a combination of shear and tensile failure. In addition, the ejection velocity distribution of the fragmented concrete mass on free surface is examined. The results indicate that the ejection velocity declines monotonously with the increase of the distance to symmetry axis of computational model. On the wall of the prefabricated borehole, two types of fragmented concrete mass are analyzed, and bottom initiation is recommended to eject the fragmented concrete mass effectively. Moreover, an algorithm of average ejection speed is developed to effectively estimate the drill capacity of high velocity, energetic (HE) projectiles. At last, the validity of numerical simulation is verified by physical experiments.

\section{Introduction}

Deep digger weapons system, which is a nonnuclear and projectile based drilling device, provides a novel tool for commanders to attack a range of deeply buried strategic targets not now available to them $[1,2]$. It uses series of drill and blast events produced by volleys of HE projectiles in specific pattern, which are fired by missile artillery system, to deliver payload to target. In this way, the depth of these high value targets that could be absolutely destroyed can reach to $100 \mathrm{~m}$. A challenge of deep digger weapons system is mucking technique, that is, how to heave the fragmented rock mass away, so it is important to investigate the cratering process and ejection behavior in blast events.

Much attention has been paid to dynamic response of concrete-like materials under blast loading in recent years [3-6]. The influence of filling coefficient, length-to-diameter ratio, and buried depth on crater size was experimentally researched by Liu et al. [7]. Zhu [8, 9] simulated the process of rock fractured and fragmentation in crater blast with a modified principal stress failure criterion using AUTODYN code. In fact, other materials models with different failure criterions, such as TCK, RHT concrete models, could also model the dynamic response of concrete-like materials preferably. However, little attention has been paid to the failure process of concrete materials under RHT concrete model, which is widely used in recent years. In addition, high velocity concrete fragmentation was simulated using SPH/MLSPH by Rabczuk and Eibl [10]. Their study suggests that the SPHmethod is able to simulate the fracture and fragmentation of concrete slabs under contact detonation. However, the ejection velocity distribution of fragmented concrete mass which is essential to estimate drill depth for deep digger weapons system has not been discussed in existing literatures [10-12].

In this paper, with the default RHT concrete model employed in AUTODYN 2D code [13], a meshfree computational model is developed to investigate the failure and ejection behavior of concrete materials under internal blast; then an algorithm of average ejection speed is developed to effectively estimate the drill capacity of HE projectiles. This work will provide guidance and reference for designing deep digger weapons system. 


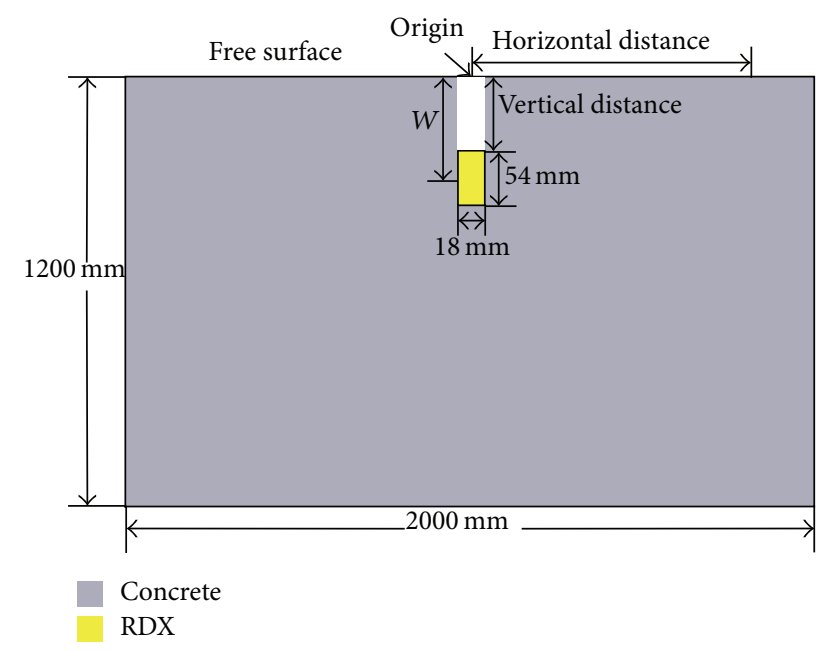

Figure 1: Computational model.

\section{Numerical Simulation}

2.1. A Cylindrical Model. Figure 1 shows a concrete body with a single borehole containing charge RDX. The geometry and loading both are axially symmetric about the borehole axis, and a 2D axial symmetrical model is developed for the numerical simulation. In this model, the radius of RDX is $9 \mathrm{~mm}$, and the buried depth is $W$. The RHT concrete model is employed, and the SPH processor is applied. Considering the dimensions of charge, the computational zone is set as $\Phi 2000 \times 1200 \mathrm{~mm}$.

2.2. Material Model. Concrete always shows typical nonlinear elasticity due to its heterogeneity and porosity. In this paper, a porous equation of state is employed. It includes three different phases to model the damage process of porous materials: elastic deformation, compaction, and solidification. During the compaction phase, pores in the material collapse. In the final solidification phase, the material is approximately homogeneous, and the volumetric response is once again elastic.

RHT concrete model is widely used by many researchers to model the dynamic response of concrete subjected to internal blast [14-16]. Three shear strength surfaces are employed in this concrete model: elastic limit surface, failure surface, and residual surface. The failure surface is defined as follows:

$$
Y_{f}^{*}=Y_{\mathrm{TXC}}^{*}(p) \cdot R_{3}(\theta, p) \cdot r_{f}(\dot{\varepsilon}),
$$

where $Y_{\mathrm{TXC}}^{*}(p)$ is the normalized compressive meridian and $r_{f}(\dot{\varepsilon})$ is the rate enhancement factor. $R_{3}(\theta, p)$ is a scalar function of lode angle $\theta$ and hydrostatic pressure $p$ and is used to describe reduced strength on shear and tensile meridian.

After being completely destroyed (damage factor $D=1$ ), the materials still have some capacity to support shear and positive stress as a result of friction in the particulate matter. At this point, the residual surface is defined as

$$
Y_{r}^{*}=B \times\left(p^{*}\right)^{M},
$$

where $B$ and $M$ are material constant and $p^{*}$ is the normalized hydrostatic pressure.

The effect of damage is modeled as a loss in deviatoric strength by interpolating between the failure and residual surface, so the maximum stress state that the materials can reach to is

$$
Y_{\text {damage }}^{*}=(1-D) Y_{f}^{*}+D Y_{r}^{*}
$$

The default tensile failure criterion of RHT for concrete in AUTODYN code is the minimum pressure failure. The minimum tensile pressure in the material is limited to

$$
p=\min \left[(1-D) \times p_{\min }, p(\rho)\right],
$$

where $p(\rho)$ is the pressure determined by equation of state for materials and $p_{\min }$ is the minimum pressure included in failure criterion.

\section{Failure Behavior of Concrete}

3.1. Fracturing Process. The pattern of damage in the process of concrete fractured at typical time is shown in Figure 2. According to different influence factors, the procedure could be divided into two distinct stages: (1) in the former stage (0 $489 \mu \mathrm{s})$, shock wave played an important role. Crushed zone, fractured zone, spalled zone, and elastic zone were formed in this stage as a result of propagation and reflection of stress wave in the concrete; (2) in the second stage (after $489 \mu \mathrm{s}$ ), the strength of stress wave was small enough to be neglected, and the expansion of detonation products turned to be the main factor. The foregoing crack extended to free surface further, then the fragmented concrete mass was ejected by the expansion of detonation, and crater was formed at last. It is worth noting that if the buried depth of charge continues to increase, the spalled zone is likely to disappear and none of the concrete material will be ejected.

3.2. Failure Mechanism. In order to investigate the failure process, it is necessary to examine the pressure loaded on concrete materials. In the explosion center, the overpressure produced by initiated explosion was on the order of $10 \mathrm{Gpa}$; then the concrete materials suffered a bunch of strong compression wave. Figure 3 shows the profile of pressure at $186 \mu \mathrm{s}$, and the pressure imposed upon target $\mathrm{E}$ is pictured in Figure 4. As can be seen from the pictures, the peak of compression wave at target $\mathrm{E}$ reduced to about 3.1 Mpa for the reason of energy dissipation. Subsequently, the materials in target E were loaded by tensile wave produced by the expansion of high pressure concrete materials. After $489 \mu \mathrm{s}$, the pressure loading became too small to destroy the concrete materials, and damaged zones tended to be completely formed.

Crushed Zone. The failure zone near the explosion was mainly fractured by shear stress. The curves of equivalent stress, failure stress, and damage versus time are presented in Figure 5. Before the time of $21 \mu \mathrm{s}$, the material was in elastic state as equivalent stress was always smaller than failure stress. At the time of $32 \mu$ s though the equivalent stress 

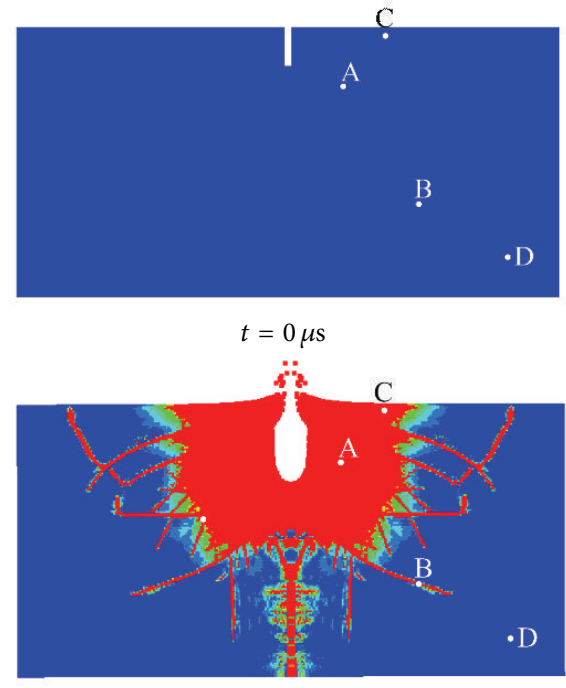

$t=489 \mu \mathrm{s}$
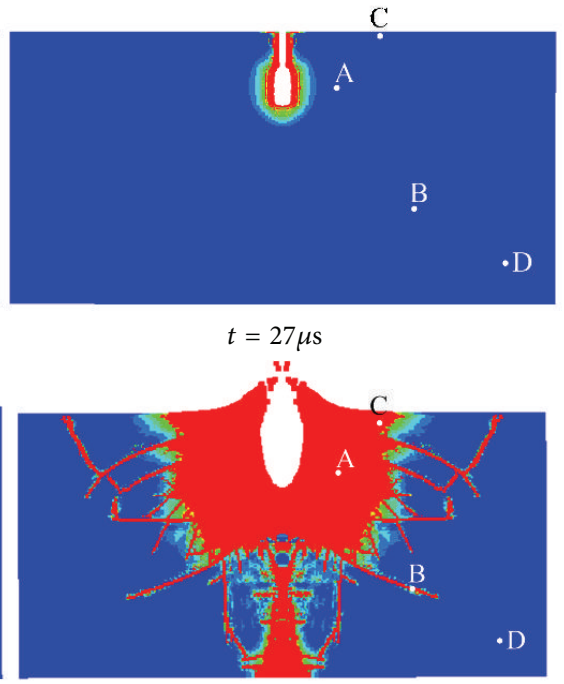

$t=1260 \mu \mathrm{s}$

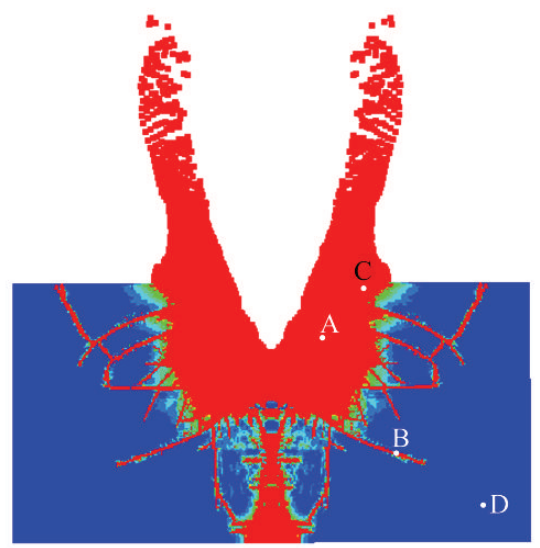

$t=10 \mu \mathrm{s}$

FIgURE 2: The pattern of damage in the process of concrete fractured.

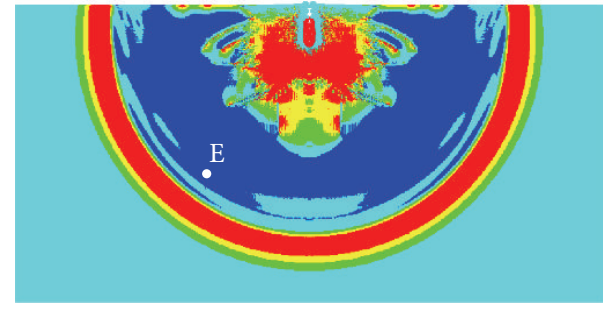

FIGURE 3: The profile of overpressure at $186 \mu$ s.

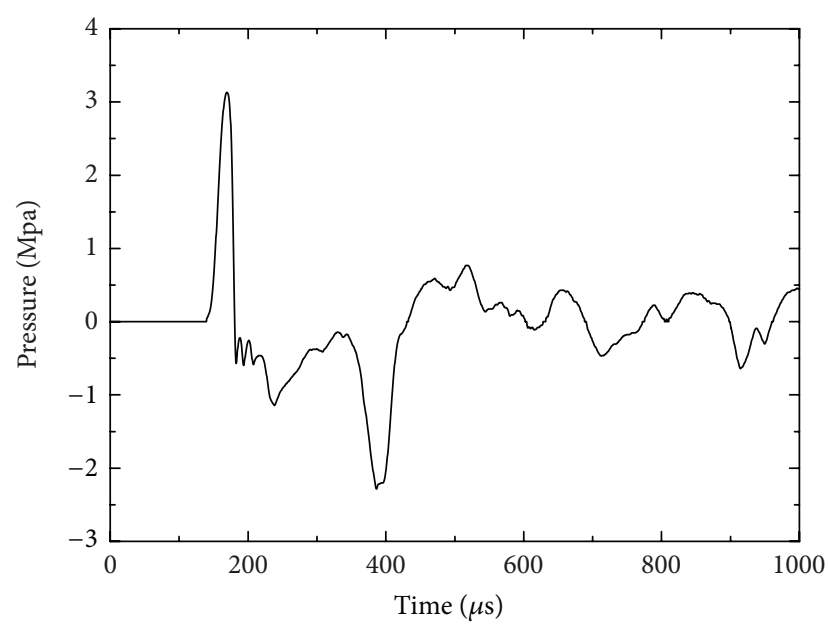

Figure 4: The pressure imposed upon target E.

reached to failure surface the damage did not tend to increase, because the failure stress was strengthened by the increased pressure. At the time of $52 \mu \mathrm{s}$, the equivalent stress reached to failure surface once again. The damage factor increased significantly as shear failure had occurred. According to (3),

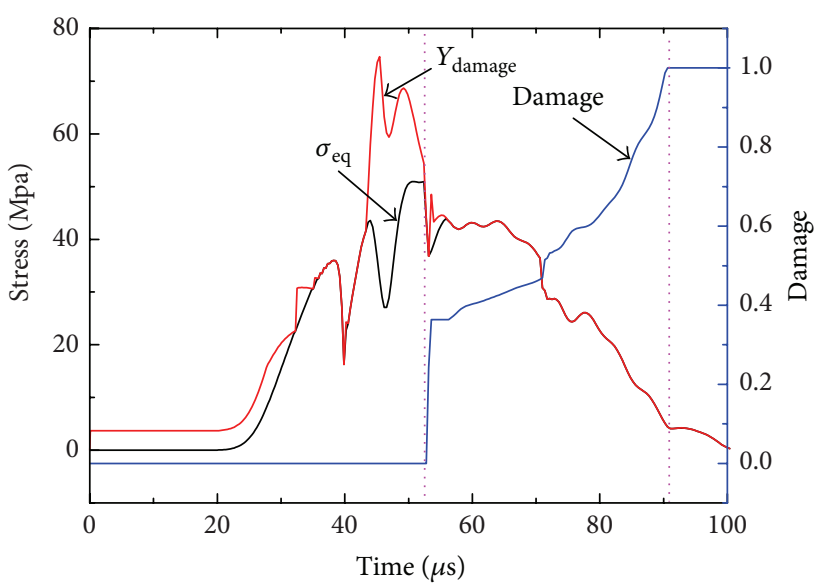

FIGURE 5: Equivalent stress, failure stress, and damage versus time of target A.

the failure surface of damaged materials is dependent on the damage factor. When the damage has been accumulated, the maximum stress that the materials can reach to gradually declines. Consequently, the curve of equivalent stress coincided with failure stress during softening process. At the time of $91 \mu \mathrm{s}$, materials in target A were completely destroyed when the stress state reached to the residual surface. However, the materials still supported shear and positive stress as a result of friction between fragmented concrete masses.

Fractured Zone. The failure zone including target B tended to fail in tensile stress. The strength of the stress wave decayed with the increased spread distance after initial detonation. The instantaneous pressure, failure pressure, and damage of target $\mathrm{B}$ are shown in Figure 6. From the curve of damage, failure started at the time of $140 \mu \mathrm{s}$ when the pressure was $-3.01 \mathrm{Mpa}$. This pressure, which was smaller than 


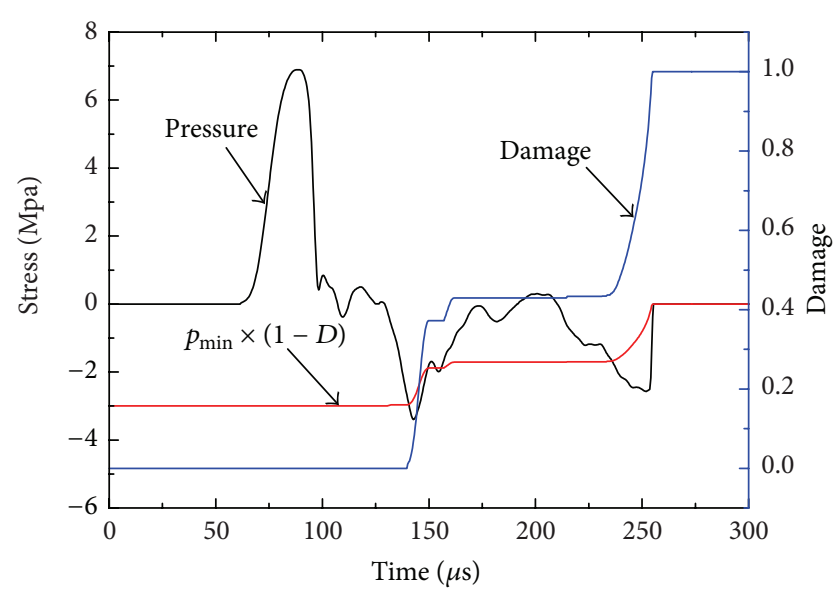

FIGURE 6: Instantaneous pressure, failure pressure, and damage versus time of target $B$.

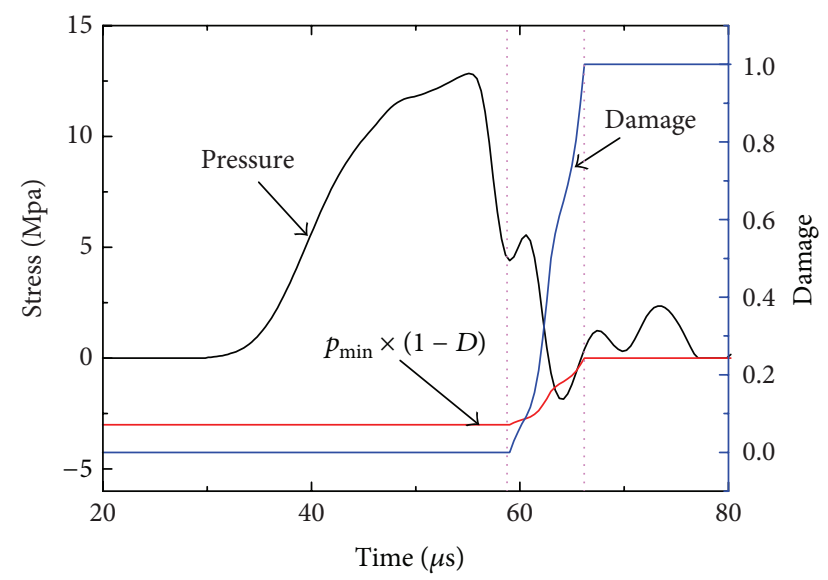

Figure 7: Pressure and damage versus time of target C.

the minimum failure pressure $-3.0 \mathrm{Mpa}$, enabled the materials to fail in tensile stress. Subsequently, the damage did not accumulate any longer as the negative pressure became larger than the minimum failure pressure. At the time of $234 \mu$ s the pressure went below the minimum failure pressure again, and the damage factor of target $B$ increased up to 1 . Therefore, concrete materials at target $\mathrm{B}$ tended to fail in tensile stress in the entire blast event.

Spalled Zone. The failure zone including target $\mathrm{C}$ near free surface was caused by a combination of shear and tensile failure. Figure 7 presents the failure process of target C. The materials encountered the compression waves coming from the center of explosion and tensile waves generated by the reflection of compression waves at free surface. The damage started to increase at the time of $58 \mu \mathrm{s}$. However, the pressure at this moment was positive (4.5 Mpa). This indicated that the materials did not fail in tensile stress. So shear failure had occurred at that moment. At the time of $63 \mu$ s, tensile failure occurred as the pressure of target $\mathrm{C}$ lied below the current

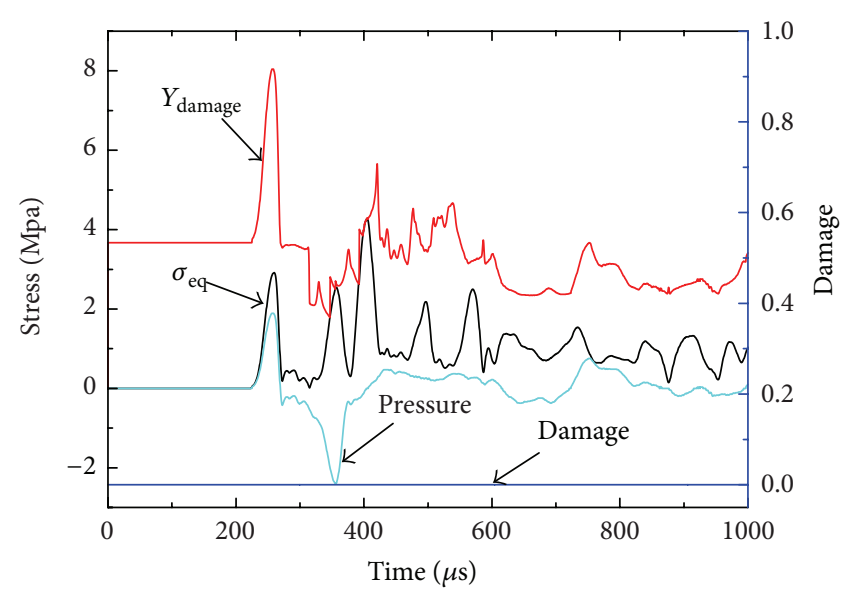

FIGURE 8: Equivalent stress, failure stress, and damage versus time of target $\mathrm{D}$.

failure pressure. At the time of $66 \mu$ s, the materials in target $C$ were completely destroyed while the damage factor is up to 1 .

Elastic Zone. In the area including target D far away from explosion center, the materials were never destroyed. Figure 8 presents the equivalent stress, failure stress, and damage versus time of target $D$. The equivalent stress of target $D$ was always smaller than the failure stress. As a result, the damage in this zone never increased and only elastic deformation had appeared in the concrete materials.

\section{Ejection Behavior of the Fragmented Concrete Mass}

4.1. Ejection Mechanism and Ejection Velocity Distribution. The energy released from the explosion in concrete can be divided into four parts: the kinetic and potential energy of detonation products, the energy consumed by fracturing, the kinetic energy of fragmented concrete mass, and the energy transformed to internal energy of concrete. The kinetic energy of fragmented concrete mass can be quantified by ejection velocity. In order to describe the distribution of ejection velocity, a Cartesian coordinate system is established. Origin and horizontal and vertical distance are defined in Figure 1. The curves of ejection velocity for typical position on free surface and prefabricated borehole are illustrated, respectively, in Figures 9 and 10.

On the free surface, the ejection velocity of fragmented concrete mass declined monotonously with the increase of the distance to symmetry axis of borehole. Figure 9 shows ejection velocity versus time at typical point on the free surface. In the point $9 \mathrm{~mm}$ away from the origin, two prominent sharp rising stages, corresponding to the effect of stress wave and the expansion of detonation products, respectively, were observed, whereas only one remarkable stage was observed at the point $160 \mathrm{~mm}$ away from the origin, which suggested that the expansion of detonation products hardly accelerates the materials far away from the center of explosion. On free 


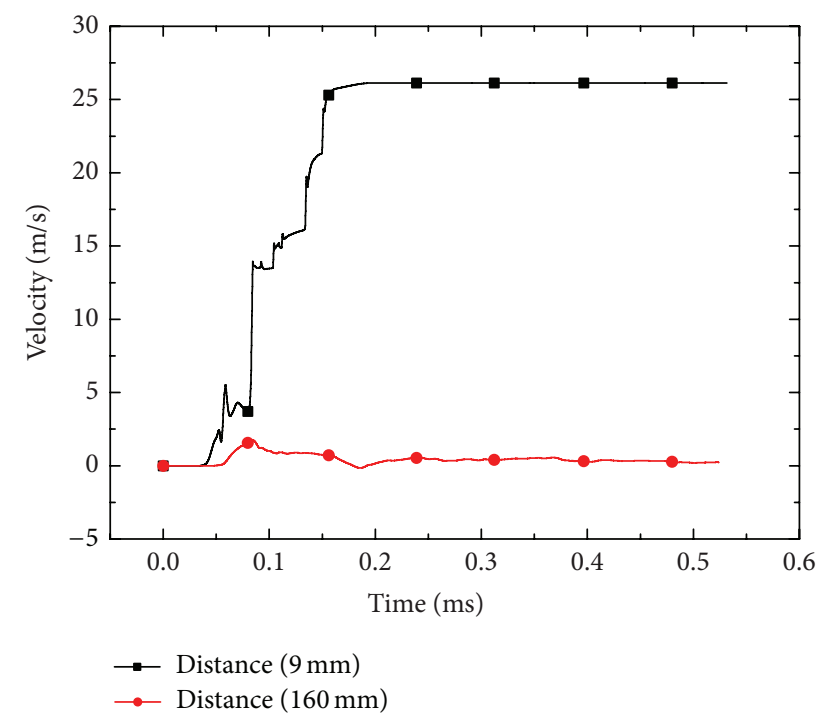

FIGURE 9: Ejection velocity versus time at typical target on the free surface.

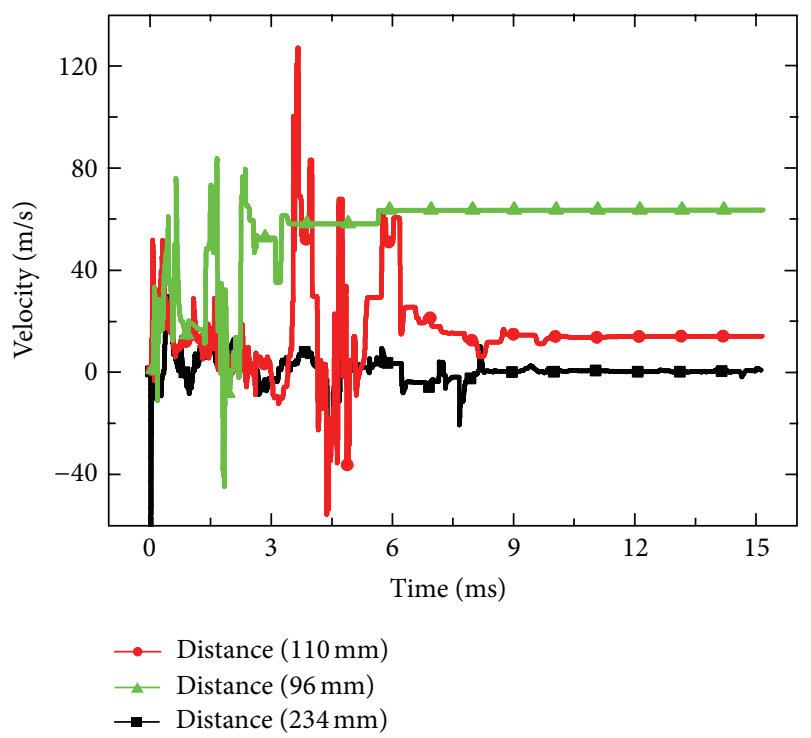

FIGURE 10: Ejection velocity versus time at typical target on the wall of borehole.

surface, materials near the opening of the borehole got the maximum ejection velocity (the value was $25.8 \mathrm{~m} / \mathrm{s}$ ).

On the wall of the prefabricated borehole, the ejection velocity was significantly vibrating as synthetically affected by shock wave and detonation products, and it had no monotonicity after becoming steady. Two types of fragmented concrete mass were found as the directions of their ejection velocity were opposite. Above the charge, such as the fragments $90 \mathrm{~mm}$ and $110 \mathrm{~mm}$ away from the origin, the directions of their velocity were upward. As can be seen from Figure 10, the velocity of fragmented concrete mass $110 \mathrm{~mm}$ away from the origin was $126 \mathrm{~m} / \mathrm{s}$ at the time of $3.6 \mathrm{~ms}$. This exactly high velocity has been reached because a small part of materials on the wall of the borehole was taken away by

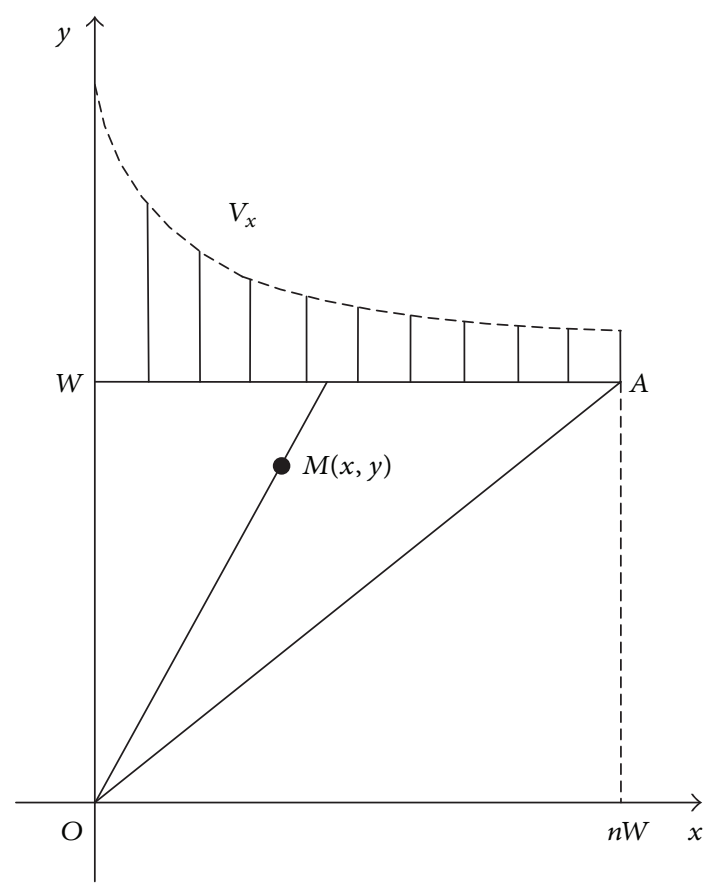

FIGURE 11: Distribution of ejection velocity of fragmented concrete mass.

the detonation products moving at even faster speed. Near the bottom of charge, such as the fragments $234 \mathrm{~mm}$ away from origin, the directions of their velocity were downward, because initiation point was set at the top of charge and materials near the charge were affected by the downward moving detonation products. Their speed dropped close to zero after impacting against undamaged materials, so they stayed in the crater till the end. Therefore bottom initiation may be a better way to eject the fragmented concrete mass effectively by decreasing the downward moving detonation products.

4.2. Average Ejection Speed. It is important to define an average ejection speed to effectively estimate the drill capacity of HE projectiles. Figure 11 describes the distribution of ejection velocity of fragmented concrete mass. In Figure 11, blasting acting index is $n=r / W$, where $r$ is circle radius of the bottom of crater and $W$ is buried depth of charge. $\triangle O A W$ represents cross section of the crater formed by internal blast, and shadow is used to describe the speed of fragments on free surface. Considering the symmetrical characteristic, we define an average ejection speed as follows:

$$
V_{\mathrm{av}}=\frac{\iint_{S_{\mathrm{OAW}}} V_{M} d x d y}{\iint_{S_{\mathrm{OAW}}} d x d y} .
$$

In the direction of $W A$, the speed distribution tends to obey exponential law [17]:

$$
V_{x}=V_{\max } \cdot \exp (-\beta x),
$$

where $x$ represents distance between a point on WA and prefabricated borehole; $V_{\max }$ is ejection speed of $W$ which is the maximum speed on the direction of $W A ; \beta$ is coefficient. 
In this paper, an invariable ejection speed is assumed along the direction of $O M$. Therefore the ejection speed of arbitrary point $M$ contained in $\triangle O A W$ is

$$
V_{M}=V_{x}^{\prime}=V_{\max } \cdot \exp \left(-\beta W \cdot \frac{x}{y}\right)
$$

where $V_{M}$ is speed of $M$ and $V_{x}^{\prime}$ is speed of the intersection of $O M$ and $W A$.

The average speed is obtained when substituting (7) to (5):

$$
\begin{aligned}
V_{\mathrm{av}} & =\frac{\iint_{S_{\mathrm{OAW}}} V_{\max } \cdot \exp (-\beta \cdot W \cdot x / y) d x d y}{\iint_{S_{\text {OAW }}} d x d y} \\
& =\frac{V_{\max }[1-\exp (-n W \beta)]}{n W \beta} .
\end{aligned}
$$

\section{Validity of Numerical Simulation}

The experimental study on the dynamic response of concrete subjected to internal blast was conducted [18]. In that study the mass of charge was $25 \mathrm{~g}$, and the buried depth was set as $200 \mathrm{~mm}$. The farthest fragment was about $50 \mathrm{~m}$ away from the center of explosion. With the air resistance neglected, the law between the ejection distance and ejection velocity of fragmented concrete mass is $L=V^{2} / g$. The ejection velocity calculated from this formula is $V=22.36 \mathrm{~m} / \mathrm{s}$. Because the quantity of the fragmented concrete mass on the wall of the prefabricated borehole is small enough to be considered, we insist that materials near the opening of the borehole get the maximum ejection velocity. So the result of numerical simulation is $15.38 \%$ larger than that of experiment. This small error could be caused by different shapes of charge. As a whole, numerical simulation shows a good consistency with physical experiment, and the validity of numerical simulation is verified.

\section{Conclusions}

A blast model was developed to investigate the failure and ejection mechanism of concrete materials under internal blast. The results showed that the concrete materials encountered the stress wave and detonation products successively. The duration of stress wave was $489 \mu$ s while that of the expansion of detonation products was more than $10000 \mu \mathrm{s}$. Failure mechanism of three typical damaged zones was investigated. The crushed zone was caused by shear failure while the fractured zone was induced by tensile failure, and the spalled zone was formed by the combination of shear and tensile failure. In addition, the ejection velocity of fragmented concrete mass was examined. Comparing with top initiation, bottom initiation may be a better way to eject the fragmented concrete mass effectively by decreasing the downward moving detonation products.

Compared with other processors, the meshfree SPH processor can simulate ejection behavior of the fragmented concrete mass successfully, as grossly distorted elements need not be deleted to ensure a sustainable solution procedure.
RHT concrete model could simulate the failure and ejection behavior preferably as numerical simulation has showed a good consistency with physical experiment.

\section{Conflict of Interests}

The authors declare that there is no conflict of interests regarding the publication of this paper.

\section{References}

[1] D. Burns, "Deep digger weapons system concept," in Proceedings of the Medium Caliber Weapons Systems Branch, US Army ARDEC, 41st Annual NDIA Gun and Missile Systems Conference, Sacramento, Calif, USA, March 2006.

[2] D. Burns, "Deep digger weapons system development," in Proceedings of the 41st Annual NDIA Gun and Missile Systems Conference, Medium Caliber Weapons Systems Branch, US Army ARDEC, Sacramento, Calif, USA, March 2006.

[3] W. Wang, D. Zhang, F. Lu, S.-C. Wang, and F. Tang, "Experimental study and numerical simulation of the damage mode of a square reinforced concrete slab under close-in explosion," Engineering Failure Analysis, vol. 27, pp. 41-51, 2013.

[4] L. B. Jayasinghe, D. P. Thambiratnam, N. Perera, and J. H. A. R. Jayasooriya, "Blast response and failure analysis of pile foundations subjected to surface explosion," Engineering Failure Analysis, vol. 39, pp. 41-54, 2014.

[5] G. Wang and S. Zhang, "Damage prediction of concrete gravity dams subjected to underwater explosion shock loading," Engineering Failure Analysis, vol. 39, pp. 72-91, 2014.

[6] W. Wang, R. Liu, and B. Wu, "Analysis of a bridge collapsed by an accidental blast loads," Engineering Failure Analysis, vol. 36, pp. 353-361, 2014.

[7] Y. Liu, Z. Duan, F. Huang, and X. Wang, "Damage effects of explosion of shelled explosive in concrete," Defence Science Journal, vol. 60, no. 6, pp. 672-677, 2010.

[8] Z. Zhu, "Numerical prediction of crater blasting and bench blasting," International Journal of Rock Mechanics and Mining Sciences, vol. 46, no. 6, pp. 1088-1096, 2009.

[9] Z. Zhu, B. Mohanty, and H. Xie, "Numerical investigation of blasting-induced crack initiation and propagation in rocks," International Journal of Rock Mechanics and Mining Sciences, vol. 44, no. 3, pp. 412-424, 2007.

[10] T. Rabczuk and J. Eibl, "Simulation of high velocity concrete fragmentation using SPH/MLSPH," International Journal for Numerical Methods in Engineering, vol. 56, no. 10, pp. 1421-1444, 2003.

[11] B. Luccioni, D. Ambrosini, G. Nurick, and I. Snyman, "Craters produced by underground explosions," Computers \& Structures, vol. 87, no. 21-22, pp. 1366-1373, 2009.

[12] Z. Tan, W. Zhang, C. Cho, and X. Han, "Failure mechanisms of concrete slab-soil double-layer structure subjected to underground explosion," Shock Waves, vol. 24, no. 5, pp. 545-551, 2014.

[13] A. Autodyn, Interactive Non-Linear Dynamic Analysis Software Version 13, User's Manual, SAS IP Inc., 2010.

[14] Z. Tu and Y. Lu, "Modifications of RHT material model for improved numerical simulation of dynamic response of concrete," International Journal of Impact Engineering, vol. 37, no. 10, pp. 1072-1082, 2010.

[15] Z. Tu and Y. Lu, "Evaluation of typical concrete material models used in hydrocodes for high dynamic response simulations," 
International Journal of Impact Engineering, vol. 36, no. 1, pp. 132-146, 2009.

[16] Y.-Q. Ding, W.-H. Tang, R.-Q. Zhang, and X.-W. Ran, “Determination and validation of parameters for Riedel-HiermaierThoma concrete model," Defence Science Journal, vol. 63, no. 5, pp. 524-530, 2013.

[17] R. Liang, "Ejection velocity of rock in cast blasting," Engineering Journal of Wuhan University, vol. 3, pp. 39-46, 1980 (Chinese).

[18] Q. Zhang, "Numerical simulation of cast velocity of rock under explosion of spherical charge," Journal of China Coal Society, vol. 18, no. 5, pp. 27-32, 1993 (Chinese). 


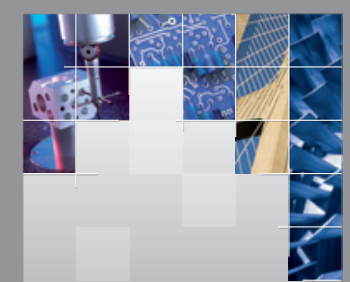

\section{Enfincering}
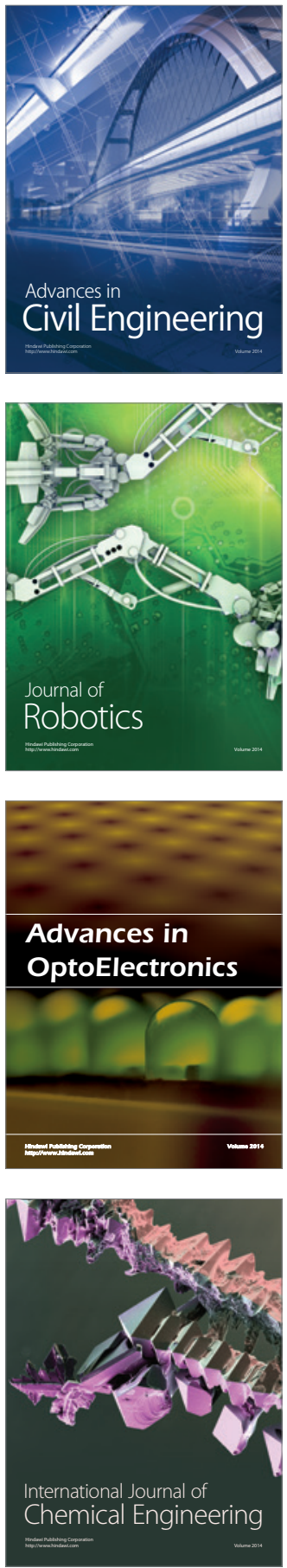

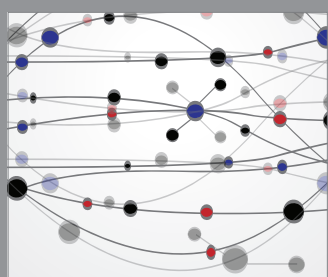

The Scientific World Journal

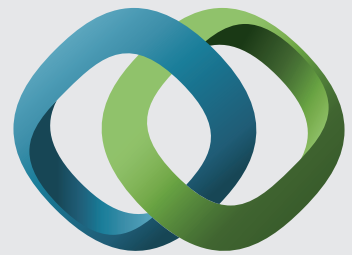

\section{Hindawi}

Submit your manuscripts at

http://www.hindawi.com
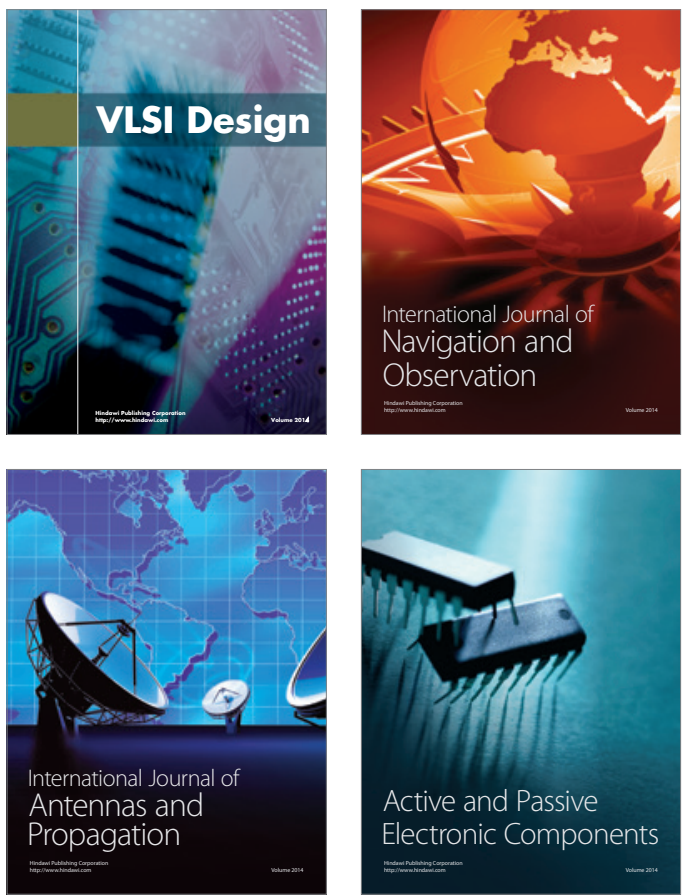
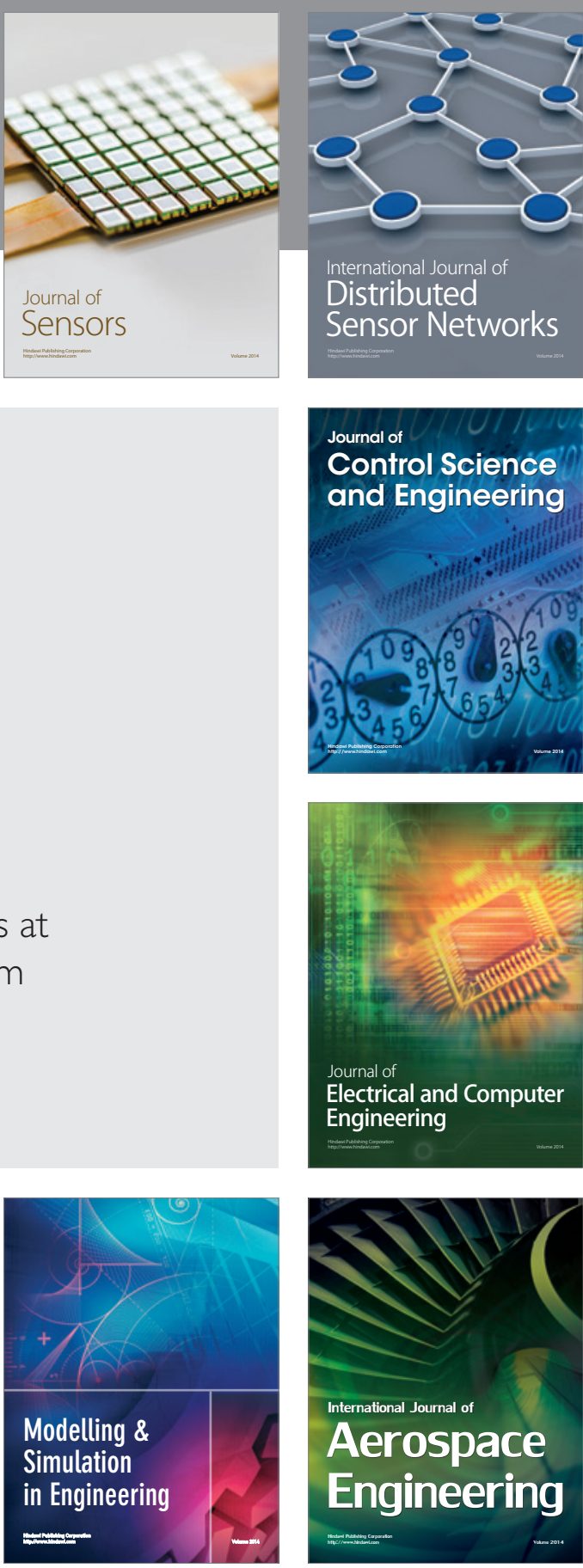

International Journal of

Distributed

Sensor Networks

Journal of

Control Science

and Engineering
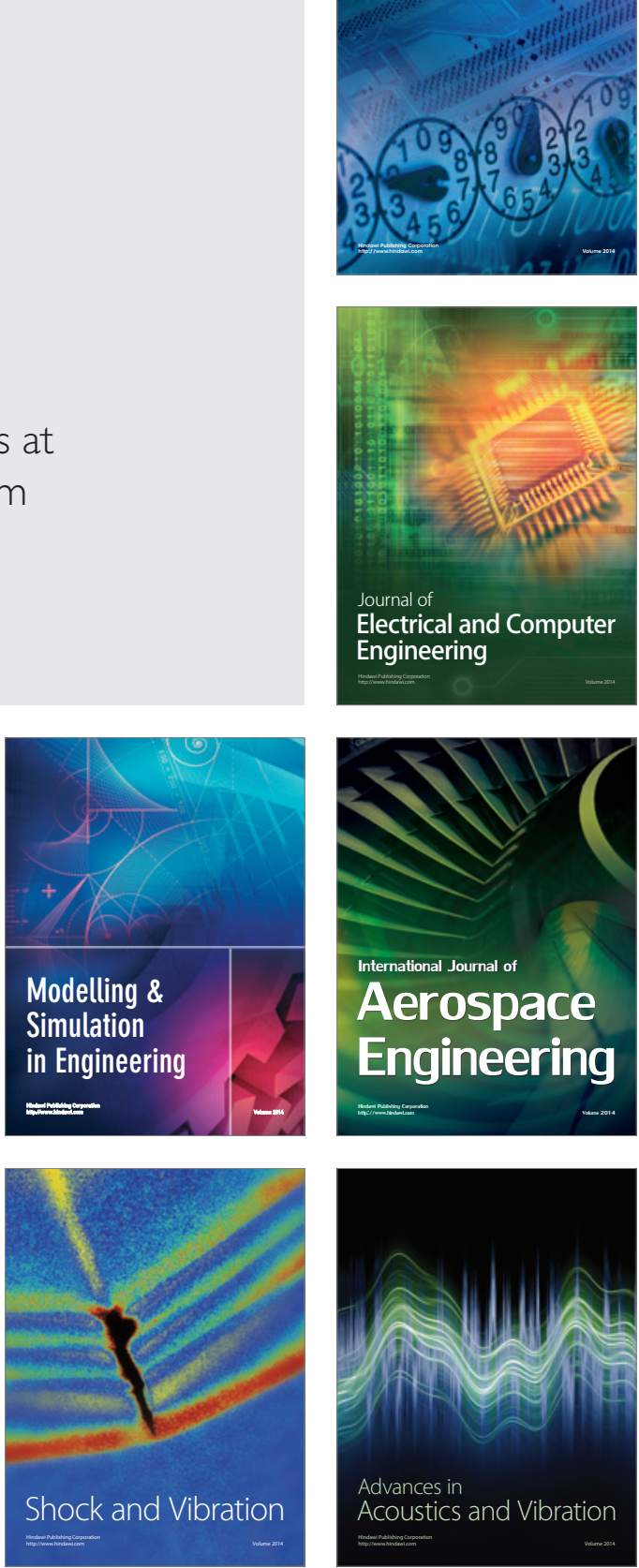